\title{
On the ergodicity of the Weyl sums cocycle
}

by

\section{BASSAM FAYAD (Paris)}

1. Let $\mathbb{T}^{2}$ denote the torus $\mathbb{R}^{2} / \mathbb{Z}^{2}$. For $\theta \in[0,1]$ define the map (skew shift) $T_{\theta}$ :

$$
\mathbb{T}^{2} \rightarrow \mathbb{T}^{2}, \quad(x, y) \mapsto(x+\theta, y+2 x+\theta),
$$

and the skew product $f_{\theta}$ :

$$
\mathbb{T}^{2} \times \mathbb{C} \rightarrow \mathbb{T}^{2} \times \mathbb{C}, \quad(x, y, z) \mapsto(x+\theta, y+2 x+\theta, z+e(y)),
$$

where $e(y)$ is the usual notation for $e^{2 \pi i y}$. The diffeomorphism $f_{\theta}$ preserves the product measure $\mu=m \times \nu$ where $m$ denotes the Haar measure on $\mathbb{T}^{2}$ and $\nu$ denotes the Lebesgue measure on $\mathbb{C}$. We say that $f_{\theta}$ is ergodic if for every $\mu$-measurable set $A \subset \mathbb{T}^{2} \times \mathbb{C}$ such that $f_{\theta}(A)=A$ we have $\mu(A)=0$ or $\mu\left(A^{\mathrm{c}}\right)=0$.

Definition 1. We define $\mathcal{F}$ to be the set of numbers $\theta \in[0,1] \backslash \mathbb{Q}$ having a continued fraction representation

$$
\theta=\frac{1}{a_{1}+\frac{1}{a_{2}+\frac{1}{\ldots}}}
$$

such that $\sum_{n} 1 / a_{n}<\infty$ and $\liminf _{q \geq 1} q^{3+\varepsilon}\|q \theta\|=0$ for some $\varepsilon>0$. Here and in all the text $\|\cdot\|$ stands for the closest distance of a real number to the integers. Let $p_{l} / q_{l}=\left[a_{1}, \ldots, a_{l}\right]=1 /\left(a_{1}+1 /\left(a_{2}+\cdots+\left(1+1 / a_{l}\right) \ldots\right)\right)$, with $p_{l}$ and $q_{l}$ relatively prime. The sequence $p_{l} / q_{l}$ is called the sequence of the best rational approximations of $\theta$ since $\left\|q_{l-1} \theta\right\| \leq\|k \theta\|$ for every $k<q_{l}$. The sequence $q_{l}$ is simply called the sequence of approximation denominators of $\theta$.

We will elaborate on the paper by Forrest [6] to obtain the following result:

TheOREm 1. Let $\theta \in \mathcal{F}$. Then $f_{\theta}$ is ergodic.

2000 Mathematics Subject Classification: Primary 11L15, 37A45; Secondary 11K60, $37 \mathrm{~A} 20$. 
The set $\mathcal{F}$ has zero measure due to any of the two conditions imposed on $\theta$. It has positive Hausdorff dimension but the condition $\sum 1 / a_{n}<\infty$ on $\theta$ is actually very restrictive since it involves all the convergents of $\theta$. For instance $\mathcal{F}$ is contained in the complement of a residual set (this can be checked by the ergodicity of the Gauss transformation $\theta \mapsto\{1 / \theta\}$ ). But we can show using a classical general argument of Halmos, presented in his introductory book on ergodic theory [3, proof of the second category theorem], that the set of $\theta$ such that $f_{\theta}$ is ergodic is a $G_{\delta}$ set, call it $\widetilde{\mathcal{F}}$. Since $\mathcal{F}$ is dense and $\mathcal{F} \subset \widetilde{\mathcal{F}}$, we have

COROLlary 1. The set $\widetilde{\mathcal{F}} \subset[0,1]$ of $\theta$ such that $f_{\theta}$ is ergodic is a residual set of positive Hausdorff dimension.

This actually hints at the possibility of bypassing the condition $\sum 1 / a_{n}$ $<\infty$ in the proof of ergodicity. Proposition 3 and hence Proposition 1, which are the only places where this condition appears, can actually be proven without it using recent results on theta sums. This will be done in a future publication.

2. Theorem 1 and its corollary strengthen the main result of [6] where the density in $\mathbb{C}$ of the Weyl sums

$$
\sum_{k=0}^{n-1} e\left(k^{2} \theta+k x\right), \quad n=1,2, \ldots,
$$

was proved for $\theta \in \mathcal{F}$ and almost every $x \in[0,1]$. Indeed, we have

Corollary 2. Let $\theta \in \widetilde{\mathcal{F}}$. Then the set

$$
B(\theta)=\left\{x \in[0,1]: \sum_{k=0}^{n-1} e\left(k^{2} \theta+k x\right), n=1,2, \ldots, \text { is dense in } \mathbb{C}\right\}
$$

is a dense $G_{\delta}$ set of full Lebesgue measure in $[0,1]$.

Proof. If $f_{\theta}$ is ergodic then for $\mu$-a.e. $u=(x, y, z)$ the sequence $u, f_{\theta}(u)$, $f_{\theta}^{2}(u), \ldots$ is dense in $\mathbb{T}^{2} \times \mathbb{C}$. This general fact can be proved by considering a countable base $\left\{O_{j}\right\}_{j \in \mathbb{N}}$ of open balls in $\mathbb{T}^{2} \times \mathbb{C}$ and observing that the complement of the invariant set $\bigcup_{n \in \mathbb{Z}} f_{\theta}^{n}\left(O_{j}\right)$ has zero measure, hence so does the complement of the set $\mathcal{D}=\bigcap_{j \in \mathbb{N}} \bigcup_{n \in \mathbb{Z}} f_{\theta}^{n}\left(O_{j}\right)$. But by definition each point $x \in \mathcal{D}$ has a dense orbit under $f_{\theta}$. Now

$$
f_{\theta}^{n}(x, y, z)=\left(T_{\theta}^{n}(x, y), z+\sum_{k=0}^{n-1} e\left(k^{2} \theta+2 k x+y\right)\right),
$$

so that for $\mu$-a.e. $(x, y, z)$ the sequence $z+\sum_{k=0}^{n-1} e\left(k^{2} \theta+2 k x+y\right), n=1,2, \ldots$, is dense in $\mathbb{C}$. This density clearly does not depend on $y$ and $z$, and the measure statement of the corollary follows. Further, $\mathcal{D}$ is a $G_{\delta}$ set and since 
its complement has zero measure it follows that it is a dense $G_{\delta}$. For the same reason, $B(\theta)$ is a dense $G_{\delta}$ set.

We will see that in proving the density of the Weyl sums (1) for almost every $x$ when $\theta \in \mathcal{F}$, Forrest actually went a long way towards proving the ergodicity of $f_{\theta}$. Yet, he left this question unsolved and put it as an open problem even for a single value of $\theta$. In a sense, we will finish his work here.

Finally, we recall that prior to [6], Forrest had already proved in [5] the transitivity of $f_{\theta}$ under the sole hypothesis $\liminf _{q \geq 1} q^{3 / 2}\|q \theta\|<\infty$. From the transitivity of $f_{\theta}$, the density of the Weyl sums follows for a dense $G_{\delta}$ set of $x \in[0,1]$. Although $T_{\theta}$ is uniquely ergodic, the cocycles $\sum_{k=0}^{n-1} e\left(k^{2} \theta+\right.$ $2 k x+y)$ behave differently for different points $(x, y) \in \mathbb{T}^{2}$ as shown by the following remark:

REMARK 1. While it is not clear whether 0 could be in $B(\theta)$ for some choice of $\theta\left({ }^{1}\right)$, it does follow from an argument by Besicovitch [2] that for any $\theta$ there always exists an $x$ such that $x \notin B(\theta)$.

3. The question of whether the set of $\theta$ for which $f_{\theta}$ is ergodic (or even transitive) has full measure (or contains all irrationals!) is still open and we do not have much to say about this, as explained in the following list of remarks:

REMARK 2. It does not seem to be known whether there exists a class of irrational numbers $\theta$ for which the Weyl sums could fail to be dense for all $x$. In [6] it is claimed erroneously $\left({ }^{2}\right)$ that the estimate $\left|\sum_{k=0}^{n-1} e\left(k^{2} \theta+k x\right)\right| \geq$ $c_{\theta} \sqrt{n}$ (uniformly in $x \in[0,1]$ ) was proved in [4] for constant type numbers $\theta$ (numbers with bounded partial quotients, or equivalently numbers that satisfy $\lim \inf _{q \geq 1} q\|q \theta\|>0$ ). If this however turns out to be true, it would obviously preclude, if $\theta$ is of constant type, the density of the Weyl sums for any choice of $x$.

Remarkably, if true, the latter estimate turns out to be paradoxically helpful in showing ergodicity of the Weyl sums without the restrictive hypothesis $\sum 1 / a_{n}<\infty$. Indeed, an elegant proof of ergodicity of $f_{\theta}$ for some class of $\theta$ (included those satisfying $\lim \inf _{q \geq 1} q^{5}\|q \theta\|=0$ ) was given in [7], and is based on the alleged uniform lower bound on the Weyl sums for constant type numbers $\theta$.

$\left({ }^{1}\right)$ The claim made by Forrest that it follows from [4] that $0 \notin B(\theta)$ for any irrational $\theta$ probably stems from his misinterpretation of the formula $a(0, n)=\Omega(\sqrt{n})$ which is used in [4] (cf. $\S 4$ below) as the negation of $a(0, n)=o(\sqrt{n})$ and not as $\sqrt{n}=O(|a(0, n)|)$ like Forrest might have understood it. It is clear from the formulae for $a(0, n)$ in the case of $\theta$ rational that one can construct an irrational $\theta$ for which there exists a sequence $q_{n} \rightarrow \infty$ such that $a\left(0, q_{n}\right) \rightarrow 0$.

$\left({ }^{2}\right)$ For the same reason as in the preceding footnote. 
REMARK 3. While a property of the rational approximations of $\theta$, at least like the one used in [5], namely $\liminf q^{3 / 2}\|q \theta\|=0$, seems necessary to study the density of the Weyl sums using the dynamics of $f_{\theta}$, the condition $\sum_{n \geq 1} 1 / a_{n}<\infty$ could be removed as in [7] from the proof if for any irrational $\theta$, the measure of the sets where $\left|\sum_{k=0}^{n} e\left(k^{2} \theta+k x\right)\right|$ is small can be controlled. It would be helpful for example if one knew that for any constant $C>0$,

$$
\lim _{q \rightarrow \infty} \sup _{1 \leq p \leq q-1} \lambda\left\{x:\left|\sum_{k=0}^{q-1} e\left(k^{2} p / q+k x\right)\right| \leq C\right\}=0 .
$$

REMARK 4 . If we denote by $f_{\theta}^{(l)}$ for $l \geq 1$ the skew product $f_{\theta}^{(l)}(x, y, z)=$ $(x+\theta, y+2 x+\theta, z+e(l y))$, then the same proof of ergodicity for $\theta \in \mathcal{F}$ of $f_{\theta}^{(1)}$ implies the ergodicity of every $f_{\theta}^{(l)}$. But the set of $\theta \in[0,1]$ with the latter property is invariant under multiplication by $l$ on the circle so it has measure either 0 or 1 .

To compare with our problem, note that twist maps of the type $\mathbb{T}^{d} \times \mathbb{R}^{k} \mapsto$ $\mathbb{T}^{d} \times \mathbb{R}^{k},(x, z) \mapsto(x+\alpha, z+\varphi(x))$, with a smooth function $\varphi$ having zero average and that is not a trigonometric polynomial are always ergodic for a dense $G_{\delta}$ set of $\alpha \in \mathbb{T}^{d}$ (of zero Hausdorff dimension however) and not ergodic for a set of $\alpha$ of full measure which consists of the Diophantine vectors, that is, vectors for which there exists $N$ such that $\liminf _{q \geq 1} q^{N}\|q \alpha\|>0$.

4. In [4], Hardy and Littlewood studied the growth of $\left|\sum_{k=0}^{n-1} e\left(k^{2} \theta+k x\right)\right|$ for different values of $\theta \in[0,1]$. Using the notation $u_{n}=\Omega\left(v_{n}\right)$ for positive sequences $u_{n}$ and $v_{n}$ to mean the negation of $u_{n}=o\left(v_{n}\right)$, the principal bounds they obtained were

Theorem ([4, Theorems 2.14, 2.141, 2.18, 2.181, 2.22, 2.221]). For any irrational $\theta \in[0,1]$,

$$
\left|\sum_{k=0}^{n-1} e\left(k^{2} \theta+k x\right)\right|=o(n), \quad \text { uniformly for all values of } x .
$$

If the partial quotients $a_{n}$ in the continued fraction expansion of $\theta$ are bounded then

$$
\left|\sum_{k=0}^{n-1} e\left(k^{2} \theta+k x\right)\right|=O(\sqrt{n}), \quad \text { uniformly for all values of } x .
$$

These are optimal bounds. Indeed, for any irrational $\theta \in[0,1]$ we have

$$
\left|\sum_{k=0}^{n-1} e\left(k^{2} \theta\right)\right|=\Omega(\sqrt{n})
$$


and for every sequence $\varphi_{n}>0$ tending to 0 as $n \rightarrow \infty$, it is possible to find irrationals $\theta$ such that

$$
\left|\sum_{k=0}^{n-1} e\left(k^{2} \theta\right)\right|=\Omega\left(n \varphi_{n}\right)
$$

With the dynamical approach adopted in this paper, the first of these equations follows immediately from two classical and elementary facts in ergodic theory (see e.g. [8]): first, that $T_{\theta}$ is uniquely ergodic as soon as $\theta$ is irrational; and second, that this implies that the function $\Phi(x, y)=e(y)$, of zero average, has its Birkhoff means $(1 / n) \sum_{k=0}^{n-1} e\left(k^{2} \theta+2 k x+y\right)$ uniformly converging to zero.

It would be nice if an additional qualitative ergodic property of $T_{\theta}$ could be displayed in the case of irrationals $\theta$ with bounded partial quotients that would explain the second bound in the above theorem of Hardy and Littlewood.

5. We now proceed to the proof of Theorem 1 . In the following, $\theta$ will be a fixed irrational number in $\mathcal{F}$. For every $n \in \mathbb{N}$ and $(x, y) \in \mathbb{T}^{2}$, let

$$
a(x, y, n)=\sum_{k=0}^{n-1} e\left(k^{2} \theta+2 k x+y\right), \quad b(x, n)=\sum_{k=0}^{n-1} e(k x) .
$$

Definition 2. We say that $l \in \mathbb{C}$ is an essential value for the cocycle $a$ above $T_{\theta}$ if for any measurable set $E \subset \mathbb{T}^{2}$ such that $m(E)>0$ and for any $\nu>0$, there exists $n \in \mathbb{N}$ such that

$$
m\left(E \cap T_{\theta}^{-n} E \cap\{(x, y):|a(x, y, n)-l| \leq \nu\}\right)>0 .
$$

We say that $l \geq 0$ is an essential value for the modulus of $a$ if for any measurable set $E \subset \mathbb{T}^{2}$ such that $m(E)>0$ and for any $\nu>0$, there exists $n \in \mathbb{N}$ such that

$$
m\left(E \cap T_{\theta}^{-n} E \cap\{(x, y):|| a(x, y, n)|-l| \leq \nu\}\right)>0 .
$$

Since $|a(x, y, n)|$ does not depend on $y$ we simply denote it by $|a(x, n)|$.

A very useful general criterion for ergodicity established by K. Schmidt in [9] states that $f_{\theta}$ is ergodic if and only if any $l \in \mathbb{C}$ is an essential value for $a$ (above $T_{\theta}$ ), but due to the symmetries of the system we have the following sufficient criterion for ergodicity that we took from [7]:

LEMMA 1. If $1 / 2$ (or any other strictly positive number) is an essential value for the modulus of a then $f_{\theta}$ is ergodic.

Proof. The proof is in two parts. First, we show that $a$ has a nonzero essential value. Indeed, otherwise by [9, Lemma 3.8] (the proof of this lemma can also be found in [1, Lemma 8.4.3]), for any compact set $K \subset \mathbb{C}$ that 
does not contain 0 , there exists a measurable set $B \subset \mathbb{T}^{2}$ such that for every $n \in \mathbb{N}$,

$$
B \cap T_{\theta}^{-n} B \cap\{(x, y): a(x, y, n) \in K\}=\emptyset,
$$

which clearly contradicts the assumption of the lemma.

Next, assume that $l \neq 0$ is an essential value for $a$. For $y_{0} \in \mathbb{T}$ denote by $S_{y_{0}}$ the map of $\mathbb{T}^{2}$ onto itself given by $S_{y_{0}}(x, y)=\left(x, y+y_{0}\right)$. Then the fact that for a measurable set $B$ with $m(B)>0$ we have an $n \in \mathbb{N}$ such that

$$
m\left(S_{y_{0}} B \cap T_{\theta}^{-n}\left(S_{y_{0}} B\right) \cap\{(x, y):|a(x, y, n)-l| \leq \nu\}\right)>0,
$$

implies for the same $n$ that

$$
m\left(B \cap T_{\theta}^{-n} B \cap\left\{(x, y):\left|a(x, y, n)-l e\left(-y_{0}\right)\right| \leq \nu\right\}\right)>0,
$$

which shows that the whole circle of radius $|l|$ is included in the set of essential values of $a$. Since the set of essential values of a complex cocycle above an ergodic map is a closed subgroup of $\mathbb{C}$ (cf. [9, Lemma 3.3]), it follows that for the cocycle $a$ it is equal to $\mathbb{C}$ and hence $f_{\theta}$ is ergodic.

6. The general strategy for controlling $|a(x, n)|=\left|\sum_{k=0}^{n-1} e\left(k^{2} \theta+2 k x\right)\right|$ starts by showing that given any infinite subsequence of approximation denominators of $\theta$, and in particular along a subsequence that satisfies $q_{n}^{3+\varepsilon}\left\|q_{n} \theta\right\| \rightarrow 0$, we have $\left|a\left(x, q_{n}\right)\right| \rightarrow \infty$ for a typical value of $x$. This implies that $\left|a\left(x, m q_{n}\right)\right|$, when $m$ is not too large, can be approximated by $\left|a\left(x, q_{n}\right)\right|\left|b\left(2 q_{n} x, m\right)\right|$ and $m$ is then chosen to bring this product close to $1 / 2$. Typically, when $2 q_{n} x$ behaves like a badly approximated number, $\left|b\left(2 q_{n} x, l\right)\right|$, $l=1, \ldots, m$, contains an $O\left(1 / m^{1-\varepsilon}\right)$-dense set in $[0,1]$ (here $\varepsilon>0$ is an arbitrarily small number). If we prove that $\left|a\left(x, q_{n}\right)\right|$ is typically bounded by $q_{n}^{1 / 2+\varepsilon}$ then the $m_{n}$ we need to modulate the product $\left|a\left(x, q_{n}\right)\right|\left|b\left(2 q_{n} x, m\right)\right|$ is not larger than $q_{n}^{1 / 2+2 \varepsilon}$ and the condition $q_{n}^{3+\varepsilon}\left\|q_{n} \theta\right\| \rightarrow 0$ then appears to be the exact condition that allows the approximation formula to hold up to this value of $m$.

Finally, to show that $1 / 2$ is actually an essential value for the modulus of $a$ we compute a bound on the derivative with respect to $x$ of the product $\left|a\left(x, q_{n}\right)\right|\left|b\left(2 q_{n} x, m_{n}\right)\right|$ and show that, under the same assumption $q_{n}^{3+\varepsilon}\left\|q_{n} \theta\right\| \rightarrow 0$, the interval $I_{n}$ containing $x$ where the product is close to $1 / 2$ is sufficiently large so that $R_{\theta}^{m_{n} q_{n}}\left(I_{n}\right)$ is almost equal to $I_{n}$. This and the fact that $|a(x, y, l)|$ does not depend on $y$ will allow us to conclude.

In this scheme, the first step is the most delicate. It was proved by Forrest in [6] who based his proof on the following approximate functional equation, established by Hardy and Littlewood in [4, Theorems 2.128, 2.17]: for $0<\theta, x<1$ and $k \geq 1$,

$$
\sqrt{\theta}|a(\theta / 2, x / 2, k)|=|a(\{1 / \theta\} / 2,\{-x / \theta\} / 2,[k \theta])|+O(1)
$$


where $\{\cdot\}$ and $[\cdot]$ denote the fractional and integer parts of a number and where the constant involved in the $O(1)$ notation is absolute. Under an additional assumption on $\theta$ it is possible to apply a dynamical approach where $\theta$ is viewed as a parameter and obtain by induction from the above functional equation a lower estimate on the Weyl sums. The upshot of this approach is the following key ingredient of [6] as well as for us here:

Proposition 1 ([6, Proposition 4.3]). Suppose $\theta \in[0,1] \backslash \mathbb{Q}$ has a continued fraction representation $\left[a_{1}, a_{2}, \ldots\right]$ such that $\sum_{n} 1 / a_{n}<\infty$. Then, given any $\delta>0$ and any infinite subset $Q$ of the set of approximation denominators of $\theta$, for Lebesgue almost every $x \in[0,1]$, there exists a sequence $q_{n} \in Q$ such that $\delta / 2 \leq\left\|2 q_{n} x\right\| \leq \delta$ and $\lim _{n \rightarrow \infty}\left|a\left(x, q_{n}\right)\right|=\infty$.

For the convenience of the reader and to keep this note as self-contained as possible (modulo the functional equation (3) that is taken for granted), we include in an appendix the scheme of the proof of the above proposition, given in [6].

7. To proceed we need the following construction similar to the one made in [6]. Let $\theta \in \mathcal{F}$. Then there exists a sequence $q_{n}$ of approximation denominators of $\theta$ such that:

7.a. $q_{n}^{3+\varepsilon}\left\|q_{n} \theta\right\| \rightarrow 0$.

7.b. For almost every $x \in[0,1]$ there is a sequence $U_{n} \rightarrow \infty$ and infinitely many $n$ such that $\delta / 2 \leq\left\|2 q_{n} x\right\| \leq \delta$ and $\left|a\left(x, q_{n}\right)\right| \geq U_{n}$ (this is exactly Proposition 1).

7.c. For almost every $x \in[0,1]$, there is an $n_{1}$ such that for $n \geq n_{1}$, we have $\left|a\left(x, q_{n}\right)\right| \leq q_{n}^{1 / 2+\varepsilon / 10}$.

This is because $\int_{0}^{1}\left|a\left(x, q_{n}\right)\right|^{2} d x=q_{n}$ implies $\lambda\left\{x:\left|a\left(x, q_{n}\right)\right| \geq q_{n}^{1 / 2+\varepsilon / 10}\right\}$ $\leq 1 / q_{n}^{\varepsilon / 5}$; but 7.a implies that $q_{n+1} \geq q_{n}^{3}$, hence $\sum 1 / q_{n}^{\varepsilon / 5}<\infty$ and 7.c follows by the Borel-Cantelli lemma.

7.d. For almost every $x \in[0,1]$, there is an $n_{2}$ such that for $n \geq n_{2}$, the set $\left\{\left|b\left(2 q_{n} x, m\right)\right|: 0 \leq m \leq q_{n}^{1 / 2+\varepsilon / 4}\right\}$ is $1 /\left(q_{n}^{1 / 2+\varepsilon / 8}\left\|2 q_{n} x\right\|\right)$-dense in $[0,1]$.

To prove this we define $H_{n}:=q_{n}^{1 / 2+\varepsilon / 4}$. We let $A_{k}^{\varepsilon} \subset[0,1]$ be the subset of irrationals such that for each $\alpha \in A_{k}^{\varepsilon}$, and all $m \geq k$, there exists a continued fraction approximation $p / q$ for $\alpha$ such that $q \in\left[m^{1-\varepsilon / 10}, m\right]$. Since the set of numbers $\alpha$ for which there exists $C>0$ such that $q_{n+1}(\alpha) \leq q_{n}(\alpha)^{1+\varepsilon / 10}$ is of full measure, we clearly have $\lambda\left(\bigcup_{k} A_{k}^{\varepsilon}\right)=1$ and we set $\lambda\left(A_{k}^{\varepsilon}\right)=1-v(k)$. We can assume that the sequence $q_{n}$ in 7 . a can be chosen so that $\sum_{n} v\left(H_{n}\right)<\infty$. Since $\lambda\left\{x \in[0,1]: 2 q_{n} x \bmod [1] \in A_{H_{n}}^{\varepsilon}\right\}=\lambda\left(A_{H_{n}}^{\varepsilon}\right)=1-v\left(H_{n}\right)$ we deduce 
that for almost every $x \in[0,1]$, there exists $n_{2}$ such that for $n \geq n_{2}$ we have $2 q_{n} x \bmod [1] \in A_{H_{n}}^{\varepsilon}$, from which $7 . d$ follows easily.

8. Note that a simple computation (see [6, Lemma A.4]) shows that for some constant $C$ and for any $x \in[0,1], l, m \in \mathbb{N}$, we have

$$
|a(x, m l)-a(x, l) b(2 l x, m)| \leq C|a(x, l)| m^{3} l\|l \theta\|,
$$

which in the case of $q_{n}$ satisfying 7.a and $m \leq q_{n}^{1 / 2+\varepsilon / 4}$ yields

$$
\left|a\left(x, m q_{n}\right)-a\left(x, q_{n}\right) b\left(2 q_{n} x, m\right)\right| \leq C\left|a\left(x, q_{n}\right)\right| q_{n}^{-1 / 2-\varepsilon / 4},
$$

and finally, if in addition $\left|a\left(x, q_{n}\right)\right| \leq 2 q_{n}^{1 / 2+\varepsilon / 10}$, then

$$
\left|a\left(x, m q_{n}\right)-a\left(x, q_{n}\right) b\left(2 q_{n} x, m\right)\right| \leq C q_{n}^{-\varepsilon / 8} .
$$

It is in the above equations that the restrictive assumption $\lim \inf q^{3+\varepsilon}\|q \theta\|$ $=0$ is really crucial.

On the other hand, we have

$$
b\left(2 q_{n} x, m\right)=e^{i 2 \pi(m-1) q_{n} x} \sin \left(2 \pi m q_{n} x\right) / \sin \left(2 \pi q_{n} x\right) .
$$

Hence for $\delta / 4 \leq\left\|2 q_{n} x\right\| \leq 2 \delta$ we have

$$
\left|b\left(2 q_{n} x, m\right)\right| \leq 1 / \delta \quad \text { and } \quad\left|D_{x}\left(b\left(2 q_{n} x, m\right)\right)\right| \leq 4 \pi m q_{n} / \delta,
$$

where $D_{x}$ denotes the derivative with respect to $x$. Also, we clearly have $\left|a\left(x, q_{n}\right)\right| \leq q_{n}$ and $\left|D_{x}\left(a\left(x, q_{n}\right)\right)\right| \leq 2 \pi q_{n}^{2}$. From these observations we conclude that for $n$ sufficiently large, for any $m \leq q_{n}^{1 / 2+\varepsilon / 4}$ and $\delta / 4 \leq\left\|2 q_{n} x\right\| \leq$ $2 \delta$, we have

$$
\left|D_{x}\left[a\left(x, q_{n}\right) b\left(2 q_{n} x, m\right)\right]\right| \leq \frac{5 \pi}{\delta} q_{n}^{2+1 / 2+\varepsilon / 4} .
$$

We deduce from 7.a to 7.d the following:

Proposition 2. Let $\theta \in \mathcal{F}$. For almost every $x \in[0,1]$ there exists an infinite sequence of integers $M_{n}$ and a sequence $\epsilon_{n} \rightarrow 0$ such that

(i) $\left\|M_{n} \theta\right\| \leq q_{n}^{-(2+1 / 2+3 \varepsilon / 4)}$;

(ii) for every $\widetilde{x} \in\left[x-q_{n}^{-(2+1 / 2+\varepsilon / 2)}, x+q_{n}^{-(2+1 / 2+\varepsilon / 2)}\right]$, we have

$$
|| a\left(\widetilde{x}, M_{n}\right)|-1 / 2| \leq \epsilon_{n}
$$

(iii) $\left\|M_{n}^{2} \theta+2 M_{n} x\right\| \leq \epsilon_{n}$.

Proof. Take a sequence $q_{n}$ satisfying 7.a. Pick an $x$ that satisfies 7.b, 7.c and 7.d. Up to taking a subsequence of $q_{n}$ we have $\delta / 2 \leq\left\|2 q_{n} x\right\| \leq \delta$ and $\left|a\left(x, q_{n}\right)\right| \rightarrow \infty$. From 7.c and 7.d, we find $m_{n} \leq q_{n}^{1 / 2+\varepsilon / 4}$ such that $\left|a\left(x, q_{n}\right) b\left(2 q_{n} x, m_{n}\right)\right| \rightarrow 1 / 2$. Since (4) is satisfied by $x$ and $m_{n}$, (ii) follows for the particular value $\widetilde{x}=x$ if we take $M_{n}:=m_{n} q_{n}$.

For $|\widetilde{x}-x| \leq q_{n}^{-(2+1 / 2+\varepsilon / 2)}$ we have $\delta / 4 \leq\left\|2 q_{n} \widetilde{x}\right\| \leq 2 \delta$, and since $\left|D_{x}\left(a\left(\widetilde{x}, q_{n}\right)\right)\right| \leq 2 \pi q_{n}^{2}$, we deduce from 7 .c that $\left|a\left(\widetilde{x}, q_{n}\right)\right| \leq 2 q_{n}^{1 / 2+\varepsilon / 10}$, hence 
(4) holds for $\widetilde{x}$ and for the same $m_{n}$ as above. Finally, (ii) then follows from (5).

From 7.a we get (i) and the fact that $\left\|M_{n}^{2} \theta\right\| \rightarrow 0$. Finally, the combination of $\left|a\left(x, q_{n}\right)\right| \rightarrow \infty$ and $\left|a\left(x, q_{n}\right) b\left(2 q_{n} x, m_{n}\right)\right| \rightarrow 1 / 2$ forces $\left|b\left(2 q_{n} x, m_{n}\right)\right|$ $\rightarrow 0$, hence $\left\|2 M_{n} x\right\|=\left\|2 m_{n} q_{n} x\right\| \rightarrow 0$ and (iii) is proved.

REMARK 5. It would be possible to ensure that $\left|a\left(x, q_{n}\right) b\left(2 q_{n} x, m_{n}\right)\right|$ stays close to $1 / 2$ on larger intervals than in (ii), which would allow relaxing the requirement (i) and hence relaxing the arithmetic condition 7.a on $\theta$. But this condition, as we saw, is optimal if we want to ensure (4), without which the product $\left|a\left(x, q_{n}\right) b\left(2 q_{n} x, m_{n}\right)\right|$ is no more interesting for our purposes.

9. Proof of Theorem 1. From Lemma 1 it is enough to prove that $1 / 2$ is an essential value for the modulus of $a$.

We will use $\lambda$ and $m$ to denote respectively the Haar measures on the tori $\mathbb{T}^{1}$ and $\mathbb{T}^{2}$. Fix $E \subset \mathbb{T}^{2}$ such that $m(E)>0$. Fix then a square $A=$ $I \times J=\left[x_{1}, x_{2}\right] \times\left[y_{1}, y_{2}\right],\left|x_{2}-x_{1}\right|=\left|y_{2}-y_{1}\right|=l>0$, such that $m(E \cap A) \geq$ $(9 / 10) m(A)$. We denote by $m_{E \cap A}$ the induced measure: $m_{E \cap A}(B)=m(E \cap$ $A \cap B)$ for any Lebesgue measurable set $B \subset \mathbb{T}^{2}$. We denote by $\pi^{*} m_{E \cap A}$ the projected measure given by $\pi^{*} m_{E \cap A}(K)=m_{E \cap A}\left(K \times \mathbb{T}^{1}\right)$ for any Lebesgue measurable set $K \subset \mathbb{T}^{1}$. Clearly, $\pi^{*} m_{E \cap A} \leq \lambda$, while $\pi^{*} m_{E \cap A}(I) \geq(9 / 10) l^{2}$, and $\lambda(I)=l$. Hence, considering the Radon-Nikodym derivative of $\pi_{*} m_{E \cap A}$ with respect to $\lambda$, we find that there exists $r_{0}>0$ and a set $\widetilde{I} \subset I$ with $\lambda(\widetilde{I})>0$ such that for any $r \leq r_{0}$ and for any $x \in \widetilde{I}$ we have $\pi_{*} m_{E \cap A}([x-$ $r, x+r]) \geq(4 / 5) l 2 r$, that is,

$$
m(\Delta(x, r) \cap E) \geq \frac{4}{5} m(\Delta(x, r)),
$$

where $\Delta(x, r)=[x-r, x+r] \times J$.

Since $\lambda(\widetilde{I})>0$, it is possible to take $x_{0} \in \widetilde{I}$ for which the statement of Proposition 2 holds. Recall that for $(x, y) \in \mathbb{T}^{2}$ and $p \in \mathbb{N}$ we write $|a(x, y, p)|$ or $|a(x, p)|$ since the modulus of $a$ does not depend on $y$. If we set $\Delta_{n}=\Delta\left(x_{0}, q_{n}^{-(2+1 / 2+\varepsilon / 2)}\right)$, Proposition $2($ ii) yields

$$
|| a\left(x, y, M_{n}\right)|-1 / 2| \leq \epsilon_{n} \quad \text { for all }(x, y) \in \Delta_{n} .
$$

From the definition of $\widetilde{I}$ we have, for $n$ sufficiently large,

$$
m\left(\Delta_{n} \cap E\right) \geq \frac{4}{5} m\left(\Delta_{n}\right) .
$$

On the other hand, (i) and (iii) imply that

$$
\lim _{n \rightarrow \infty} \frac{m\left(T_{\theta}^{-M_{n}} \Delta_{n} \Delta \Delta_{n}\right)}{m\left(\Delta_{n}\right)}=0,
$$

where $\triangle$ stands for symmetric difference. 
It follows immediately from (7) and (8) (because $4 / 5>1 / 2$ ) that for $n$ sufficiently large,

$$
m\left(T_{\theta}^{-M_{n}} E \cap E \cap \Delta_{n}\right)>0,
$$

and (6) then implies that $1 / 2$ is an essential value for the modulus of $a$.

Appendix: Proof of Proposition 1. We sketch the proof given in [6]. For the bound on $\left\|2 q_{n} x\right\|$ note that for any strictly increasing sequence of integers $l_{n}$, the set of $x$ such that the sequence $\left(l_{n} x\right)_{n \in \mathbb{N}}$ is dense has full Lebesgue measure. Hence we just have to show that for any infinite subset $Q$ of the set of approximation denominators of $\theta$, for Lebesgue almost every $x \in[0,1]$, there exists a sequence $q_{n} \in Q$ such that $\lim \left|a\left(x, q_{n}\right)\right|=\infty$.

First, it is easy to see that the set of $x \in[0,1]$ satisfying the above condition is invariant under translation by $\theta$; but $x \mapsto x+\theta$ is ergodic, hence it is enough to prove that the set in question has positive measure. Next, by a simple computation, for a given $k \in \mathbb{N}$ and any sequence $q_{n}$ such that $q_{n}\left\|q_{n} \theta\right\| \rightarrow 0$, we obtain

$$
2 \max \left\{\left|a\left(x+k \theta, q_{n}\right)\right|,\left|a\left(x, q_{n}\right)\right|\right\} \geq\left\|2 q_{n} x\right\||a(x, k)|+C_{k} u_{n}
$$

where $u_{n} \rightarrow 0$ as $n \rightarrow \infty$ (cf. [6, Corollary A.5]). Hence the proof is reduced to the following

Proposition 3 ([6, Proposition 3.13]). Suppose $\theta \in[0,1] \backslash \mathbb{Q}$ has a continued fraction representation $\left[a_{1}, a_{2}, \ldots\right]$ such that $\sum_{n} 1 / a_{n}<\infty$. Then there is a $\varrho>0$ such that for all $C>0$, there is a $k$ such that $\lambda\{x$ : $|a(x, k)| \geq C\} \geq \varrho$.

To prove Proposition 3, it is convenient to define first the following function similar to the modulus of the Weyl sums:

$$
\psi(\theta, x, k):=\left|\sum_{j=0}^{k-1} e\left(j^{2} \theta / 2+j x\right)\right| .
$$

Then for $\theta>0$ and $x<1$,

$$
\sqrt{\theta} \psi(\theta, x, k)=\psi(\{1 / \theta\},\{-x / \theta\},[k \theta])+O(1)
$$

where $\{\cdot\}$ and $[\cdot]$ denote the fractional and integer parts and the constant in $O(1)$ is absolute. Equation (9) is the only "hard analysis" estimate that is needed in [6], but it is really crucial since it is at the center of the proof of Proposition 3. It was obtained by Hardy and Littlewood [4, 2.128, 2.17] as a generalisation of a formula of Lindelöf in the case of $\theta$ rational and its proof is based on the calculus of residues.

We now explain how (9) is used to prove Proposition 3. Given $k \in \mathbb{N}$, let $S \theta=\{1 / \theta\}$ and write $\widetilde{S}(\theta, x)=(S \theta,\{-x / \theta\})$ and $\left(S^{m} \theta, U_{\theta}^{(m)} x\right)=$ 
$\widetilde{S}^{m}(\theta, x)$. Let $\sigma_{m}(\theta)=\sqrt{S^{m-1} \theta} \sigma_{m-1}(\theta)$ with $\sigma_{0}(\theta)=1$, and $k(m)=$ $\left[k(m-1) S^{m-1}(\theta)\right]$ with $k(0)=k$. By induction from (9) we have

$$
\sigma_{m}(\theta) \psi(\theta, x, k)=\psi\left(S^{m} \theta, U_{\theta}^{(m)} x, k(m)\right)+O(1)
$$

(the constant in $O(1)$ is absolute and comes from $O\left(\sum_{l=1}^{m} \sigma_{m-l}\left(S^{l} \theta\right)\right)=$ $O\left(\sum_{l=1}^{m} 2^{-l / 2}\right)$ since the hypothesis $\sum 1 / a_{n}<\infty$ implies $\lim \sup a_{n} \geq 2$, which in turn shows that $\sigma_{j}\left(S^{p} \theta\right) \leq C(\theta) 2^{-j / 2}$ for any $p$ and $j$ ).

Recall the notation $b(x, k)=\sum_{j=0}^{m-1} e(j x)$. Since $\psi(\theta, x, k)=|b(x, k)|+$ $O\left(k^{3}\|\theta\|\right)$ with an absolute constant in the error term, from (10) we have

$$
\sigma_{m}(\theta) \psi(\theta, x, k) \geq\left|b\left(U_{\theta}^{(m)} x, k(m)\right)\right|-C\left(k(m)^{3}\left\|S^{m} \theta\right\|+1\right)
$$

for some absolute constant $C$. On the other hand, the condition $\sum 1 / a_{n}<\infty$ is crucial (see [6, Corollary 3.6]) in checking that for all $0<\eta<1 / 2$ and all $m \geq 1$,

$$
\lambda\left\{x:\left\|U_{\theta}^{(m)} x\right\|<\eta\right\} \geq \widetilde{C} \eta \quad \text { for some absolute constant } \widetilde{C},
$$

which by an elementary computation implies that for any $C_{0} \geq 1$,

$$
\lambda\left\{x:\left|b\left(U_{\theta}^{(m)} x,\left[2 \pi C_{0}\right]+1\right)\right| \geq C_{0}\right\} \geq \widetilde{C} /\left(2\left[2 \pi C_{0}\right]+2\right) .
$$

Fix now $C_{0} \geq 3 C$ where $C$ is the constant of (11). Given any $C^{\prime}>0$ pick $m$ sufficiently large so that $C_{0} /\left(3 \sigma_{m}(\theta)\right) \geq C^{\prime}$ and $\left(\left[2 \pi C_{0}\right]+1\right)^{3}\left\|S^{m} \theta\right\| \leq 1$ (possible due to the arithmetical condition on $\theta$ ). Let $k=k(0)$ be such that $k(m)=\left[2 \pi C_{0}\right]+1$. From (11) we have

$$
\begin{aligned}
\left\{x: \psi(\theta, x, k) \geq C^{\prime}\right\} & \subset\left\{x: \sigma_{m}(\theta) \psi(\theta, x, k) \geq C_{0} / 3\right\} \\
& \subset\left\{x:\left|b\left(U_{\theta}^{(m)} x,\left[2 \pi C_{0}\right]+1\right)\right| \geq C_{0}\right\},
\end{aligned}
$$

and by (12) the latter set has measure greater than $\varrho=\widetilde{C} /\left(2\left[2 \pi C_{0}\right]+2\right)$.

Acknowledgments. I am grateful to Mariusz Lemańczyk, François Parreau, Jean-Paul Thouvenot and Robert Vaughan for many useful conversations, and to Mahesh Nerurkar and Dalibor Volný for communicating to me their preprint [7]. I thank the referee for his useful remarks.

\section{References}

[1] J. Aaronson, An Introduction to Infinite Ergodic Theory, Math. Surveys Monogr. 50, Amer. Math. Soc., 1997.

[2] A. Besicovitch, A problem on topological transformations of the plane, II, Proc. Cambridge Philos. Soc. 47 (1951), 38-45.

[3] P. Halmos, Lectures on Ergodic Theory, Chelsea, New York, 1960.

[4] G. Hardy and J. Littlewood, The trigonometric series associated with the elliptic $\theta$-functions, Acta Math. 37 (1914), 193-239.

[5] A. Forrest, The limit points of Weyl sums and other cocycles, J. London Math. Soc. (2) 54 (1996), 440-452. 
[6] A. Forrest, Symmetric cocycles and classical exponential sums, Colloq. Math. 84/85 (2000), 125-145.

[7] M. Nerurkar and D. Volný, On the ergodicity of Weyl sum cocycles, preprint.

[8] W. Parry, Topics in Ergodic Theory, Cambridge Univ. Press, Cambridge, 1981.

[9] K. Schmidt, Cocycles of Ergodic Transformation Groups, Lecture Notes in Math. 1, Mac Millan of India, 1977.

\section{CNRS UMR 7539}

Université Paris 13

93430 Villetaneuse, France

E-mail: fayadb@math.univ-paris13.fr

Received on 24.3.2005

and in revised form on 27.9.2006 